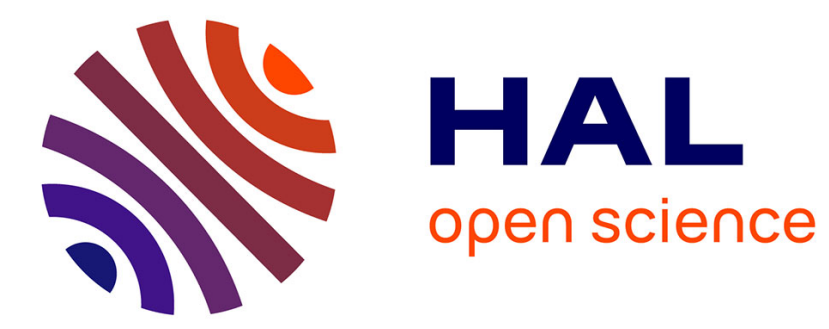

\title{
Au plus profond de soi
}

Pierre Frolla, Gilles Raveneau

\section{- To cite this version:}

Pierre Frolla, Gilles Raveneau. Au plus profond de soi. Ethnologie française, 2006, 36 (4), pp.681-683. 10.3917/ethn.064.0681 . hal-03134806

\section{HAL Id: hal-03134806 https://hal.parisnanterre.fr/hal-03134806}

Submitted on 8 Feb 2021

HAL is a multi-disciplinary open access archive for the deposit and dissemination of scientific research documents, whether they are published or not. The documents may come from teaching and research institutions in France or abroad, or from public or private research centers.
L'archive ouverte pluridisciplinaire HAL, est destinée au dépôt et à la diffusion de documents scientifiques de niveau recherche, publiés ou non, émanant des établissements d'enseignement et de recherche français ou étrangers, des laboratoires publics ou privés. 


\title{
Au plus profond de soi
}

\author{
Propos recueillis par Gilles Raveneau \\ Pierre Frolla \\ plongée libre en apnée
}

Pierre Frolla est né à Monaco en 1975 où il réside toujours aujourd'hui. C'est très jeunes que lui et son frère Philippe ont été sensibilisés à la plongée par leur père, lui-même champion d'apnée et membre de l'équipe monégasque de chasse sous-marine. Le déclic se produira en 1994 lors d'un stage organisé par Nice Inspiration. Il s'agit d'un groupe d'apnéistes issu du Nice Université Club Omnisports, dans lequel on trouve l'universitaire Claude Chapuis, Olivier Heuleu, Marc Counil, Jean-Marc Tominic et Loïc Leferme, fondateurs de l'Association internationale pour le développement de l'apnée (AIDA), qui organise le premier championnat du monde d'apnée le 4 octobre 1996 et dont Pierre Frolla est, aux côtés de son frère et de pêcheurs sous-marins de renom, un des apnéistes de sécurité.

Sa passion, son travail, sa vie, c'est la plongée en apnée. À trente et un ans, il est devenu, avec des athlètes comme Umberto Pelizzari et Loïc Leferme, l'un des plongeurs libres les plus en vue. Il pratique toutes les modalités de l'apnée ${ }^{1}$, dans chacune desquelles il excelle. Il a ainsi été triple recordman du monde en apnée libre ${ }^{2}$ de 1999 à 2001 à - 72, - 73 et - 80 mètres et est devenu la neuvième personne à franchir la barre des -100 mètres $\left(-123 \mathrm{~m}\right.$ en 2004) en no limits ${ }^{3}$. Ses records ne s'arrêtent pas là : il a également été champion de France en poids variable en 1998 et 1999 à - 82 et - 90 mètres, lauréat du Trophée européen en apnée poids constant à - 57 mètres, vainqueur en individuel et par équipe de la Freediver Open en apnée dynamique en 2003.

En dehors de ses propres entraînements, Pierre Frolla enseigne sa discipline au sein de L'École bleue à Monaco, structure qui initie les enfants à la plongée libre et à la plongée en scaphandre, tout en les sensibilisant au respect de l'environnement. Par ailleurs, il a ouvert son propre Centre École de Plongée en Apnée, dans lequel il propose des stages de découverte s'adressant aussi bien aux adultes qu'aux enfants. Depuis peu, il s'est engagé avec succès dans l'organisation d'événements sportifs ou de soutien à des œuvres caritatives.

C'est la plongée qui m'a appelé, ce n'est pas moi qui l'ai choisie. Je vis au bord de la mer à Monaco et mon père faisait partie de l'équipe de chasse sous-marine dans les années soixante. J'habiterais à Grenoble, je serais sûrement champion de ski. C'est le milieu dans lequel tu évolues qui est déterminant. Je ne connais pas d'apnéistes nés au centre de la France qui soient devenus très forts.

La plongée en apnée est tout pour moi. C'est à la fois une passion, un travail et ma vie au quotidien. Avant d'être une discipline sportive, l'apnée est une façon de vivre, un moyen de me découvrir réellement. Elle demande de se connaitre et d'apprendre à être accepté par le milieu. Il est inutile de forcer, la mer est toujours plus forte. Pour pouvoir plonger profond, il faut rentrer au plus profond de ses sensations. Ce sont des sensations de glisse, de pureté, de solitude, de plénitude avec l'eau et d'écrasement à la fois. Pour ne pas souffrir, tu dois justement te relâcher face à la pression et au manque d'oxygène. Bien sûr, les images mentales sont différentes entre une descente magique à quarante mètres où tu te laisses bercer par le courant et une compétition. Il s'agit de descendre au plus profond de soi; découvrir comment son corps fonctionne et s'adapte. Mon corps est un terrain de recherche. Cela me permet de savoir quelles sont mes limites, mes craintes et mes engouements, ce qui va bien ou non, ce que je dois faire pour atteindre tel objectif. Mais il ne s'agit pas de repousser ses limites, car tu vas toujours là où tu es capable d'aller, au-delà tu meurs. C'est très porteur comme expression, mais elle est fausse. Ce qui m'intéresse est de savoir pourquoi aujourd'hui j'ai été si mauvais alors que la veille j'atteignais telle profondeur. C'est cela le vrai sens du problème. Et la réponse est : c'est dans ta tête. Quand tu arrives à savoir comment tu fonctionnes, cela te permet aussi de mieux vivre en société. Tu peux tricher avec les gens; avec l'apnée, tu ne peux pas.

Le risque a une dimension importante car il est le vecteur de la réussite ou de l'échec de ce que tu entreprends. La plongée est une activité très risquée si elle n'est pas bien faite. Cela demande de mettre en place des systèmes de sécurité qui doivent être les meilleurs pour te permettre de descendre et de remonter en toute sécurité, ainsi qu'à ton équipe. Le risque doit donc être étudié, connu et maîtrisé. On ne joue pas avec. On n'est pas là pour flirter avec la mort. Pour cela, tu dois avoir une bonne connaissance de toi et de l'élément dans lequel tu évolues. Aujourd'hui par exemple, la mer est belle, il fait beau. Hier, on s'est entraîné avec des creux de deux mètres. On a dû s'arrêter. On avait prévu de descendre à soixante mètres, on n'est allé qu'à dix.

La manière dont on mesure le danger ici est simple. Il n'y a pas deux poids, deux mesures : soit tu descends, tu remontes et tout va bien; soit tu descends, ca ne va pas et tu meurs. Le danger, c'est la noyade et la mort. Tu peux toujours avoir des petits accidents barotraumatiques, mais ce n'est pas très grave. Si tu compares 


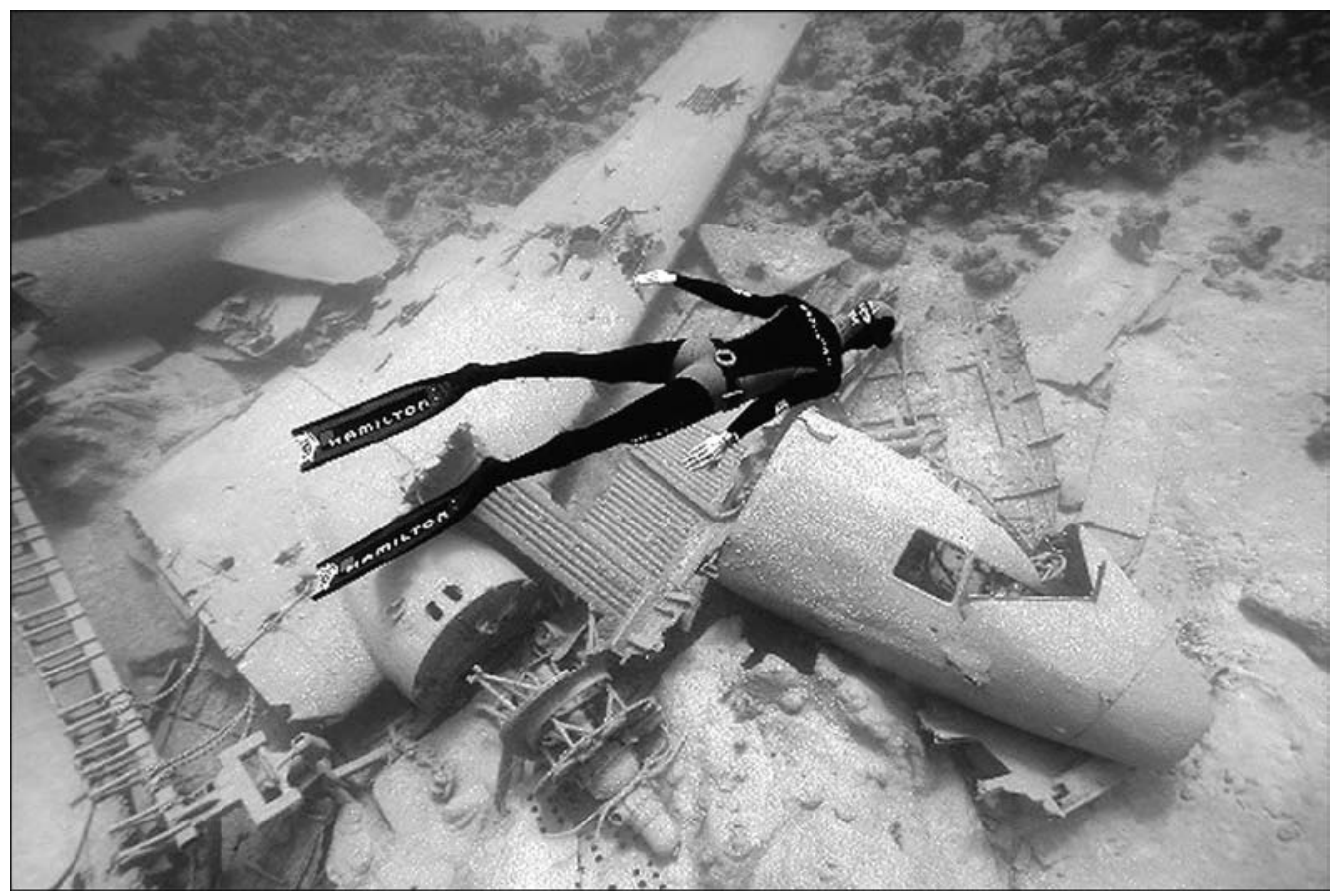

1. Épave entre 10 et 40 m, Bahamas, 2006 (photo Frédéric Buyle).

l'apnée avec une descente à ski de super-géant, tu as toute une graduation de dangers : une chute, une glissade, une fracture, une porte ratée. Dans notre discipline, c'est très sommaire : ou tout va bien ou tout va très mal. Il n'y a pas de juste milieu. C'est la raison pour laquelle l'apnée demande un cheminement très lent. Une parfaite maitrise de soi, une bonne connaissance du milieu et du matériel utilisé, et surtout un regard critique sur toi-même. Quant tu ne peux pas descendre, tu ne le fais pas. Tu ne peux pas dire: "Je vais essayer, on verra bien." Par exemple, lorsque je me préparais pour la compétition en poids variable en 1998, le record du monde était à cent quinze mètres et j'en étais à sept. On n'avait pas des créneaux d'entraînement très disponibles. Si je laissais passer une séance, il fallait que j'attende dix jours pour me réentraîner. On n'avait pas de temps à perdre. Un matin, j'arrive très fatigué et je me dis : "C'est pas grave, au pire j'arrête. " Mais quand tu es parti pour descendre, tu ne t'arrêtes jamais, tu vas jusqu'au bout. Je suis remonté et j'ai fait un œdème pulmonaire, c'est-à-dire une forme de "perfusion" de sang dans les poumons. L'œdème en apnée n'est pas fatal comme celui que tu peux faire en plongée "bouteille ", avec l'embolie gazeuse qui te monte au cerveau. Ça m'a fait mal et je n'ai pas pu plonger pendant cinq semaines... Il a fallu quand même que j'attende 2004 pour battre ce record. Voilà un exemple. Ca aurait pu être vraiment beaucoup plus grave, j'ai eu de la chance.

Mais tu ne peux pas compter sur la chance. La chance, c'est lorsque tu tombes par hasard sur une épave. Dans cette discipline sportive, elle n'existe pas. Tu le fais bien et ça passe, ou tu le fais mal et ça casse. Imagine un coureur sur un terrain de quatre cents mètres, qui se donne à fond dès le début. Au bout de trois cents mètres, il n'en peut plus et sort du couloir, c'est tout. En apnée, si tu décides de descendre à quatre-vingts, et si ça ne va pas à la remontée à cinquante, tu ne peux pas sortir. Tu es obligé de remonter jusqu'en haut! Et si tu as la malchance de rester accroché à un filet, tu y restes. Imagine que tu as des autonomies très faibles qui sont de l'ordre de dix secondes à peu près. À cent mètres, tu vas consommer onze fois plus d'oxygène qu'en surface. Donc, lorsque tu passes une seconde au fond, c'est comme si tu en passais onze en surface. Le calcul est très simple : dix secondes fois onze, cela fait cent onze, soit quasiment deux minutes. Ce n'est pas comme en escalade où tu peux avoir la chance que ton piton retienne la corde à laquelle tu es accroché quand tu tombes.

Je n'ai pas peur sous l'eau. Une fois, j'ai été vraiment effrayé : je me suis retrouvé face à un requin-tigre de six mètres cinquante. Là c'est vrai, j'ai eu la chance qu'il mette du temps à comprendre que j'étais comestible ! Il n'y a pas de raison d'avoir peur parce que tu progresses petit à petit et que tu chemines lentement. Si demain je décide de descendre à quatre-vingts, c'est parce que la veille je suis descendu à soixante-dix-huit, et l'avantveille à soixante-seize, et ainsi de suite.

J'ai conscience des dangers auxquels cette pratique expose parce que j'enseigne à des enfants dès l'âge de huit ans. La première chose que je leur apprends est de 


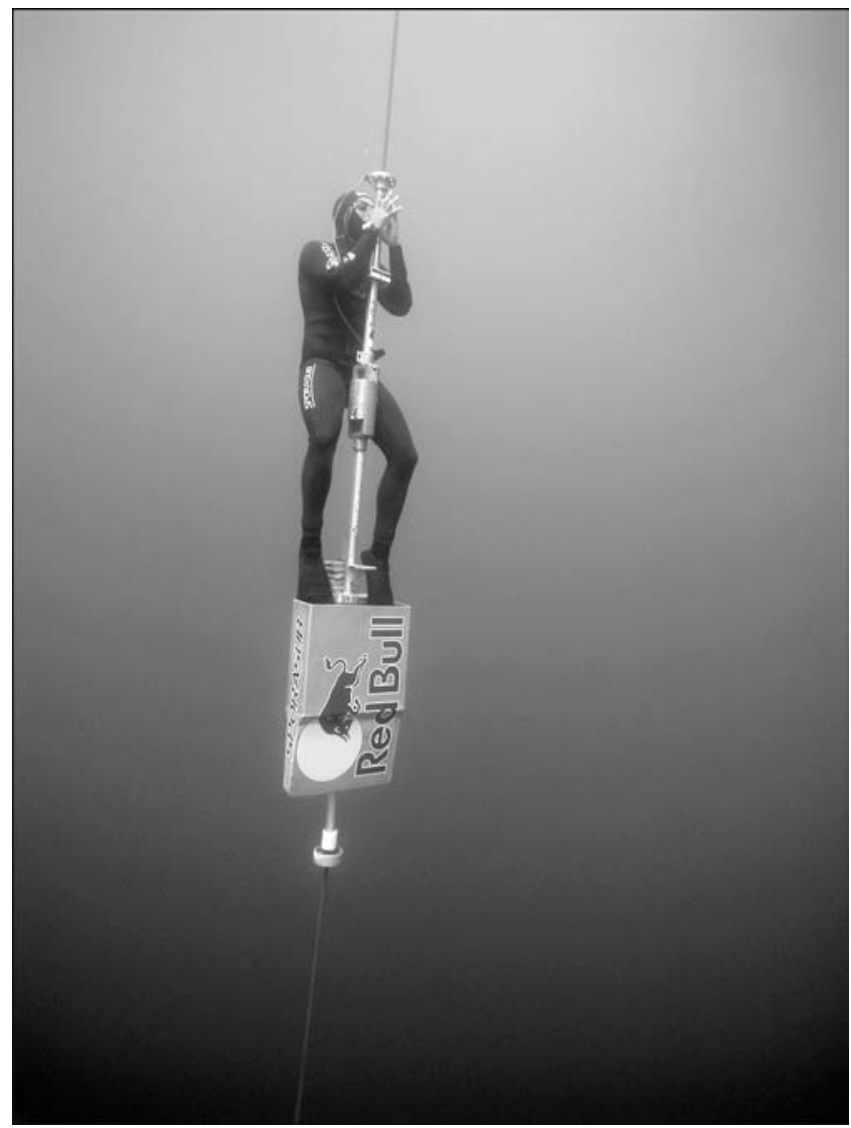

2. Descente officielle de record du monde 2004, à $123 \mathrm{~m}$ en poids variable, Bahamas, 2006 (photo Frédéric Buyle).

ne pas avoir peur de ce qu'il y a dans l'eau : les méduses, les poissons, etc. On n'a peur que de l'inconnu. Dès que tu connais, que ce soit un milieu ou toi-même, tu n'as plus peur. Par exemple, lorsque j'étais enfant et que j'étais à la pêche au thon avec mon père à quarante

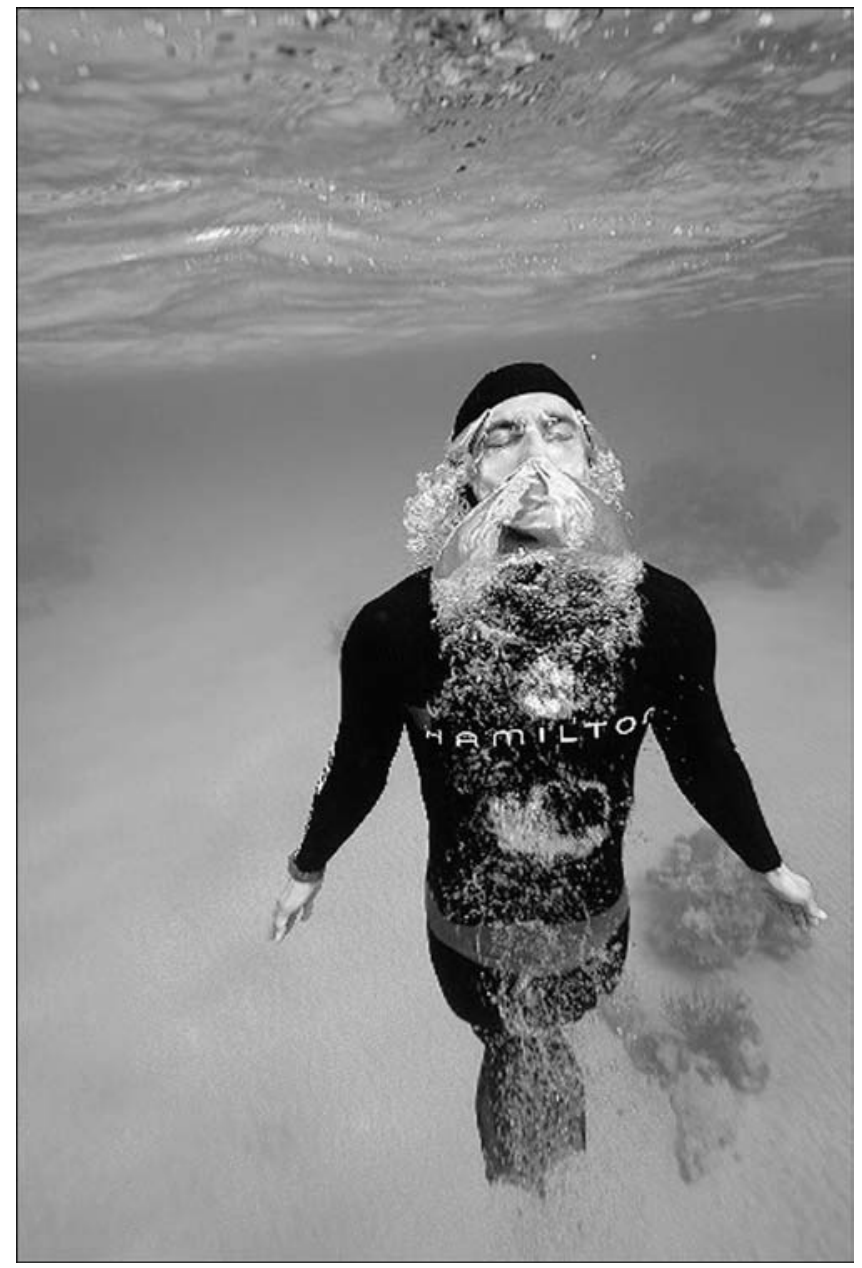

3. Remontée en surface, Bahamas, 2006 (photo Frédéric Buyle).

milles au large, j'avais peur de me baigner. J'avais cette appréhension de ne pas pouvoir voir le fond. L'apnée m'a permis de ne plus avoir peur de ça.

\section{Notes}

1. Bien que la plongée en apnée soit à la base de la pratique de la chasse sous-marine, la Fédération française d'études et de sports sousmarins (FFESSM) ne reconnaît aucun record et n'organise aucune compétition d'apnée, contrairement à l'Association internationale pour le développement de l'apnée (AIDA). Les compétitions d'apnée no limits ne sont pas reconnues par la Confédération mondiale des activités subaquatiques (CMAS), agréée par le
Comité international olympique (CIO), qui regroupe 107 pays. Cette discipline peine à se faire reconnaître et à se faire accepter par les instances fédérales. Sur le plan national, ces compétitions ne sont pas homologuées par la FFESSM, qui possède la délégation du ministère des Sports et qui privilégie l'apnée loisir et la sécurité, en limitant notamment la profondeur à 40 mètres.

2. L'apnéiste descend et remonte tout seul, sans palmes, mais en tirant avec les bras sur le câble.
3. Le plongeur descend "tracté " par un poids - la gueuse - et remonte à l'aide d'un ballon ou d'une combinaison gonflable. Cette modalité est la plus dangereuse ; les records sont aussi les plus profonds.

4. Il s'agit de descendre à l'aide d'une gueuse et de remonter à la palme et/ou en se hissant à l'aide du filin.

5. L'apnéiste s'immerge à faible profondeur, le but étant de parcourir la distance la plus importante. Cette modalité se pratique avec ou sans palmes. 